\title{
Drug-Induced Acute Angle-Closure Glaucoma: Raising your Index of Suspicion
}

\author{
Jazli Tan Jia An ${ }^{1}$, Sheikh Izzat Bin Zainal-Abidin Bahajjaj ${ }^{1}$, Fatimah Lateef ${ }^{2 *}$ and Zhang Yuan \\ Helen $^{3}$ \\ ${ }^{1}$ National University of Singapore, Yong Loo Lin School of Medicine, Singapore \\ ${ }^{2}$ Professor and Senior Consultant, Department of Emergency Medicine, Singapore
}

${ }^{3}$ Associate Consultant, Department of Emergency Medicine, Singapore

*Corresponding author: Lateef Fatimah, Department of Emergency Medicine, Singapore General Hospital, Singapore

\section{ARTICLE INFO}

Received: 慧 June 09, 2021

Published: 业 June 17, 2021

Citation: Jazli Tan Jia An, Sheikh Izzat Bin Zainal-Abidin Bahajjaj, Fatimah Lateef, Zhang Yuan Helen. Drug-Induced Acute Angle-Closure Glaucoma: Raising your Index of Suspicion. Biomed J Sci \& Tech Res 36(4)-2021. BJSTR. MS.ID.005878.

Abbreviations: AACG: Acute Angle-Closure Glaucoma; ED: Emergency Department; ACG: Angle Closure Glaucoma; IOP: Intraocular Pressure; ADR: Adverse Drug Reactions; SSRIs: Selective Serotonin Reuptake Inhibitors; SLE: Systemic Lupus Erythematosus
ABSTRACT

Aim: To highlight polypharmacy as a risk factor for acute angle-closure glaucoma (AACG) in the emergency department (ED) with a brief literature review and propose an approach to early identification of AACG in the critically ill patients.

Background: Acute angle-closure glaucoma (AACG) is an ophthalmological emergency that can lead to rapid, irreversible vision loss as a result of ischemia and atrophy of the optic nerve. Various drugs have been known to precipitate an acute angleclosure attack including adrenergics, anticholinergics, antihistamines, cholinergics, sulfonamides and serotonergic agents. Drug-induced AACG is usually observed in patients with predisposing risk factors. Anatomical risk factors include shallow chamber depth, small anterior segment and plateau iris configuration. Demographic risk factors include advanced age, family history, female gender and Asian ethnicity. Increasing rates of polypharmacy in the population, especially amongst the elderly, result in many patients with these predisposing risk factors also being on drugs that may precipitate AACG. More importantly, many drugs routinely prescribed acutely in the ED tend to overlap with the drugs reported to trigger AACG. Lastly, when an unresponsive patient presented to the ED, a diagnosis of AACG might be missed if the patient is unable to relay the classical symptoms. These three factors necessitate a high index of suspicion to ensure that a sight-threatening cause is not missed out in our patients.

Keywords: Drug-Induced Angle-Closure Glaucoma; Polypharmacy; Emergency Department

\section{Introduction}

Angle Closure Glaucoma (ACG) refers to the obstruction of aqueous outflow between the anterior and posterior chambers of the eye [1]. The resulting obstruction then leads to raised intraocular pressure (IOP), which may cause optic neuropathy as well as other visual field defects. Acute Angle Closure is important to catch in the ED as it is an ophthalmic emergency which may lead to irreversible blindness within hours or days [2]. Glaucoma is still the leading cause of blindness worldwide [3], and it is estimated that at least $1 / 3$ of AACG cases are related to prescription drugs [4]. In tertiary institutions, especially in areas such as the ICU (intensive care unit) and High Dependency wards, there are however patients who are critically ill and are often on multiple drugs who may predispose them to developing AACG but may have been missed as ophthalmological examination is not routinely performed. This paper hopes to provide insights on the prompt diagnosis of AACG especially in ED patients. 


\section{Risk Factors}

Risk factors for AACG can be separated into anatomical and demographic risk factors. Anatomical risk factors are those that lead to a crowded anterior segment, namely a shallow central anterior chamber depth, a thick and anteriorly positioned lens and a short axial length of the eye. Optical coherence tomography has been used to evaluate other anatomical risk factors including a smaller anterior chamber width, thicker and more curved irides, and a larger lens vault. Epidemiological risk factors on the other hand include the female sex, advanced age and the Asian ethnicity [5].

\section{Polypharmacy as a Risk Factor}

Polypharmacy is another important risk factor that seems to be under recognised. It refers to the use of one or more medications to treat multimorbidity chronically, and complexity emerges during acute ED presentation as the elderly are prone to drug-drug and drug-disease interactions based on physiological changes. As one ages, more diseases are accumulated, leading to more drugs being prescribed to treat these diseases and their complications. Additionally, evidence-based prescription guidelines generally rely on the findings of clinical trials that exclude older patients with multiple comorbidities. Guidelines for appropriateness of prescribing, including the Beers criteria for Inappropriate Medication Use in Older Patients, do not include guidance on combinations of multiple medications. This leaves the elderly vulnerable to drug-drug interactions and adverse drug reactions (ADR) $[5,6]$.

ADRs occur disproportionately more among the geriatric demographic even when adjusting for increased drug usage. Hospital admissions as a result of ADRs have also increased in the past 20 years, especially among those above 80 years old $[3,7]$. ADRs in the elderly commonly present as geriatric syndromes such as falls, but this paper seeks to explore AACG specifically. The later sections will analyse the various types of drugs that have been known to precipitate ACG. The overlap between these drugs and drugs that are commonly implicated in polypharmacy is significant [6].

\section{Clinical Presentation}

Symptoms of AACG classically include acute headache, ocular pain, blurring of vision, nausea, vomiting and halos around lights. On examination, possible findings are an increased intraocular pressure, a mid-dilated and unresponsive pupil, corneal edema, conjunctival hyperemia and a shallow anterior chamber [7]. Dilation of the pupil as a result of drugs, darkness or a stress response can trigger an AACG attack.

\section{Recognition in a Critically Ill Patient}

The emergency department sees numerous patients with altered mental states daily. In such patients, it may be much easier to miss a case of AACG especially if they are unable to communicate the classical symptoms mentioned earlier. One must have a high index of suspicion on physical examination of the patient's eyes especially if one notices a unilateral red eye, swollen eyelids with or without prior trauma and/or patient presents with symptoms of retching, persistent vomiting or other non-specific complaints. This should prompt the physician to examine the eyes closer, in particular using a light to look for a mid-dilated pupil and sluggish constriction in the affected eye. The physician should also look for a cloudy cornea reflective of corneal edema. A shallow anterior chamber can be assessed by shining a light at the temporal aspect of the cornea towards the root of the nose and observing for a semicircular shadow of the iris at the nasal area [8]. Note that the depth of the anterior chamber in the unaffected eye can also be assessed as chamber depth is usually equal bilaterally. In a situation where one pupil is unable to constrict, a reverse RAPD (relative afferent pupillary defect) test can be carried out to observe for signs of optic neuropathy from the ACG [9]. This test involves shining a light at the eyes, and observing for greater constriction of the normal eye during a direct response (when the light is shined directly at the normal eye) as compared to a consensual response (when the light is shined at the mid dilated glaucomatous eye).

Digital palpation can also be considered to assess intraocular pressure. If IOP (intra-ocular pressure) is very high, the orbit will feel rock hard on palpation, however pressure)r this should be performed with caution in trauma patients. Positive physical exam findings can be correlated with background information of the patient and other collateral information. For example, if the patient had just walked from a light room to a dark room, if she/ he was under significant stress, or if she/he possessed any of the epidemiological and/or pharmacological risk factors as mentioned earlier. A hypermetropic patient can be clued out based on his/ her spectacles which will have a high positive grade. The spectacle lenses will be thick and when looking through, objects will be magnified. In the ED setting, more portable investigations for measuring IOP must be utilised. These include the icare tonometer and tonopen [10]. The icare pro is able to derive the IOP at a level of accuracy closest to the Goldmann Applanation Tonometer, the gold standard for measuring IOP and widely used in the clinic setting [11]. A portable slit lamp can also be used to assess the depth of the anterior chamber in the unresponsive patient. The point-of-care ultrasound scan has limited utility in this case, though there has been a report of its use to diagnose a case of AACG secondary to a vitreous haemorrhage [12]

\section{Pathophysiology}

Glaucoma is differentiated into open-angle and closed-angle subtypes based on the mechanism of disruption in the drainage of aqueous humour. Aqueous humour is drained at the chamber angle mainly via the trabecular meshwork and the canal of Schlemm with 
minor drainage through the uveoscleral outflow. The iridocorneal angle is created by the posterior surface of the cornea and the iris. The angle of Schlemm meanwhile runs beneath the trabecular meshwork. In AACG, the iridocorneal angle is suddenly obstructed, causing a build-up of aqueous humour and a sharp increase in the intraocular pressure [13]. In contrast, the iridocorneal angle is open in open angle glaucoma and the pathology is mainly due to a disruption of the trabecular meshwork. However, the role of IOP in POAG is controversial as it may not necessarily be elevated in all patients [14]. AACG can be categorised based on the mechanism of the iridocorneal angle blockage. There are 4 mechanisms which are separated based on anatomical level; the iris, ciliary body, lens and forces posterior to the lens. Some of these mechanisms may also be present together. At the level of the iris, the most common mechanism is a pupillary block in which aqueous humour encounters resistance flowing from the posterior chamber to the anterior chamber creating a pressure gradient that causes anterior bowing of the iris. As a result the irido-corneal angle is reduced.

As for the ciliary body, abnormal positioning may result in the ciliary processes being more anterior. This forces the peripheral iris

Table 1: Below summarizes drugs that have been found to precipitate ACG in the past as well as their trade names and the mechanisms by which they precipitate the disease.

\begin{tabular}{|c|c|c|}
\hline Drug Class & Drug chemical and trade name & Mechanism \\
\hline Adrenergic agonists & $\begin{array}{c}\text { Ephedrine (Akovaz, Corphedra) } \\
\text { Phenylephrine (Neo-Synephrine, Biorphen) } \\
\text { Salbutamol (Ventolin, Albuterol) }\end{array}$ & Mydriasis and thickening of the iris base \\
\hline Anticholinergics & $\begin{array}{l}\text { Atropine (Atropen, Daturin) } \\
\text { Tropicamide (Mydriacyl) } \\
\text { Ipratropium bromide (Atrovent) }\end{array}$ & $\begin{array}{l}\text { Mydriasis results from antagonism at the } \\
\text { muscarinic receptors on the iris sphincter } \\
\text { muscle }\end{array}$ \\
\hline Antihistamines & $\begin{array}{c}\text { Promethazine (Phenergan, Phenadoz) } \\
\text { Cetirizine (Zyrtec, Aller-Tec) } \\
\text { Chlorpheneramine (Chlortrimeton) } \\
\text { Brompheniramine (Dimaphen) } \\
\text { Ranitidine (Zantac) } \\
\text { Cimetidine (Tagamet) }\end{array}$ & Mydriasis and swelling of the lens \\
\hline Cholinergics & $\begin{array}{l}\text { Pilocarpine (Dioptic, Alcon) } \\
\text { Acetylcholine (Michol E) } \\
\text { Carbachol (Miostat) }\end{array}$ & Anterior displacement of iris lens diaphragm \\
\hline Sulfonamide-containing medications & $\begin{array}{c}\text { Furosemide (Lasix) } \\
\text { Hydrochlorothiazide (Microzide, Apo-Hydro) } \\
\text { Acetazolamide (Diamox) } \\
\text { Topiramate (Topamax, Trokendi XR) } \\
\text { Trimethoprim-sulfamethoxazole (Bactrim, Co- } \\
\text { trimoxazole) } \\
\text { Sulfadiazine (Lentrisol, Neotrizine) }\end{array}$ & $\begin{array}{l}\text { Ciliary body edema and relaxation of lens } \\
\text { zonules }\end{array}$ \\
\hline
\end{tabular}

into the iridocorneal angle, narrowing the angle. This phenomenon is also referred to as plateau iris. The lens can also cause AACG in two instances. Firstly, a large lens can compress against the nearby iris and ciliary body, pushing them anteriorly and thus narrowing the iridocorneal angle. Secondly, when the lens is subluxed anteriorly. Forces posterior to the lens are seen in a type of glaucoma known as malignant glaucoma. The increased pressure gradient between the vitreous and aqueous compartments is due to misdirection of aqueous humour into the vitreous. As a result, the lens-iris diaphragm is displaced forward and there is also anterior rotation of the ciliary body. The iris is pushed against the trabecular meshwork and there is closure of the iridocorneal angle. Apart from the 4 major mechanisms listed above, there exist some miscellaneous causes. These include ciliary body cysts, ciliary body tumours or inflammation and air bubbles introduced after intraocular surgery. Peripheral anterior synechiae, a consequence of angle closure, can also be caused by iris and angle neovascularisation, iridocorneal endothelial syndrome and anterior uveitis [15].

\section{Drugs Involved in AACG}

(Table 1). 


\begin{tabular}{|c|c|c|}
\hline & Aripripazole (Abilify) & Sumatriptan (Sumavel DosePro) \\
Serotonergic agents & Venlafaxine (Effexor) & $\begin{array}{c}\text { Mydriasis and increased aqueous humour } \\
\text { production } \\
\end{array}$ \\
\hline Anticoagulants & Mirtazapine (Rameron) & Escitalopram (Lexapro) \\
\hline
\end{tabular}

\section{Adrenergic Agonists}

Alpha-adrenergic agents that are commonly used include mydriatic agents, vasoconstrictors and over-the-counter flu remedies. Mydriatics such as phenylephrine and apraclonidine are used to dilate pupils for fundus examinations by ophthalmologists and optometrists [7]. Phenylephrine eye drops induced AACG in $0.03 \%$ of non-selected patients while Lachkar et al reports observing two cases caused by apraclonidine eye drops [4]. Flu remedies also commonly contain phenylephrine, an a-1 agonist, which has vasoconstrictive properties to reduce nasal congestion. However, it also causes mydriasis by contracting iris dilator muscles. Mydriasis precipitates AACG via 2 mechanisms, thickening of the iris base causing adhesions and closure of the iridocorneal angle, and mid-dilation of the pupil which brings the lens closer to the iris, thus increasing the risk of pupil block AACG has also been precipitated by systemic administration of ephedrine for flu, surgical aneasthesia as well as epinephrine to treat anaphylaxis and ventricular fibrillation. Nasal ephedrine and naphazoline, used in the treatment of acute epistaxis, has also been reported to cause AACG, most likely due to reflux through the nasolacrimal duct [7] Commonly used beta2-adrenergic agonists include salbutamol, albuterol and terbutaline for asthma and chronic obstructive pulmonary disease. Especially when administered via the nebulised route, medication may accidentally enter the eye, resulting in ciliary muscle relaxation, mydriasis and increased production of aqueous humour. Therefore, it is important to ensure that masks and handheld nebulisers are properly fitted and positioned to minimise this inadvertent deposition of nebulised medication in the eyes [4].

\section{Anticholinergics}

Types of anticholinergics are numerous and have various uses. Some examples of anticholinergic agents commonly used include atropine and tropicamide used for mydriasis in fundus examinations. The risk of precipitating AACG after using mydriatic agents in a clinical setting is 3 in 10,000. In contrast to adrenergic, anticholinergic mydriasis results from antagonism at the muscarinic receptors on the iris sphincter muscle. The difference in mechanisms explains why the risk of AACG is amplified especially if both adrenergic and anticholinergic agents are applied to the eye [6,7]. Benztropine and trihexyphenidyl are used to treat parkinsonism, specifically dyskinesia. Studies have shown conflicting evidence on the effects of these drugs on AACG. In some studies, there was no recorded increase in IOP or AACG triggered by benztropine and trihexyphenidyl. However, there was a study that showed prolonged use of trihexyphenidyl leading to AACG occurring 1-2 years of chronic use of the drug.

Glycopyrrolate is used to decrease airway secretions and reverse neuromuscular blockades. One study reported bilateral AACG 12 hours after use of glycopyrrolate for neuromuscular blockade reversal. Ipratropium and tiotropium are used to treat obstructive pulmonary diseases such as asthma and COPD. There have been numerous studies on the synergistic effects of ipratropium and salbutamol on precipitating AACG with ipratropium causing mydriasis and salbutamol causing an increase in production of aqueous humour. Oxybutynin and tolterodine have a role in treating overactive bladders by inhibiting the M3 muscarinic receptors on detrusor muscles. Once again, the evidence is conflicting with one prospective trial finding no significant increase in IOP. However, there have been studies showing cases of both unilateral and bilateral AACG after use of oxybutynin. Scopolamine is another commonly used medication in the treatment of nausea and vomiting related to motion sickness. There have been cases of bilateral AACG after a scopolamine patch was handled prior to touching the eye or inserting contact lenses [7].

\section{Antihistamines}

Antihistamines block the $\mathrm{H} 1$ and $\mathrm{H} 2$ receptors and also possess anticholinergic properties. $\mathrm{H} 1$ receptor antagonists such as brompheniramine and chlorpheniramine are used to treat allergic reactions while $\mathrm{H} 2$ receptor antagonists such as cimetidine and ranitidine are used to treat gastroesophageal reflux and duodenal ulcers. The mechanism by which these drugs precipitate ACG is similar to that of cholinergic drugs.

\section{Cholinergics}

Cholinergic agents such as pilocarpine and acetylcholine cause miosis by stimulating the iris sphincter muscle, pulling the iris away from the trabecular meshwork. As such, pilocarpine may be useful to treat AACG by widening the iridocorneal angle and re-establishing drainage of aqueous humour [16]. Paradoxically, pilocarpine 
may induce AACG due to anterior displacement of the iris-lens diaphragm which consequently closes the iridocorneal angle. (lachkar) Eyes with zonular weakness or exfoliation syndrome have been found to be prone to this mechanism of angle closure (ritch $r$, angle closure glaucoma: clinical types). In intraocular surgery, acetylcholine and carbachol may also precipitate pupillary block in susceptible patients [17].

\section{Sulfa-Based Drugs}

The sulfa-based drugs including acetazolamide, hydrochlorothiazide, furosemide, cotrimoxazole, and topiramate have shown evidence inducing AACG $[18,19]$. Acetazolamide is used to reduce IOP, hydrochlorothiazide and furosemide used as diuretic agents to remedy fluid overload states, topiramate is an anticonvulsant and cotrimoxazole is an antibiotic often used in urinary tract infections. The mechanism by which AACG occurs in these cases is an allergic reaction to the sulfa component that results in ciliary body edema and relaxation of the lens zonules. There is also anterior displacement of the ciliary body, lens and iris, further increasing the risk of AACG [20].

\section{Serotonergic Agents}

The serotonergicagents of noteareantidepressants, sumatriptan and aripiprazole. Selective serotonin reuptake inhibitors and serotonin-norepinephrine reuptake inhibitors have been involved in cases of ACG. SSRI's increase the amount of postsynaptic serotonin, leading to increased stimulation of serotonin 5-HT7 receptors in the eye and subsequently iris sphincter relaxation. Selective serotonin reuptake inhibitor (SSRIs) of note include venlafaxine, bupropion, mirtazapine, and escitalopram. The overall result is mydriasis and increased aqueous humour production that may trigger ACG. SNRIs on the other hand increase adrenergic and dopaminergic activity, causing mydriasis as well. There exist case reports of duloxetine and venlafaxine causing ACG (20). Sumatriptan has also been reported to influence ACG $[21,22]$. The proposed mechanism is drug-induced malignant glaucoma, forward rotation of the lens-iris diaphragm, zonular relaxation and increased curvature of the lens. These factors contribute to a predisposition for ACG [22]. A proposed mechanism for ACG induced by Aripripazole is also activity at the $5 \mathrm{H}-\mathrm{T}$ receptors or supraciliary effusion induction that displaces the lens-iris diaphragm anteriorly [23].

\section{Anticoagulants}

ACG secondary to haemorrhage as a complication of anticoagulation has also been reported. These case reports have presented with vitreous, choroidal and subretinal hemorrhages. In one case report, patients with exudative age-related macular degeneration who were also on anticoagulation developed ACG after massive vitreous and subretinal hemorrhages [24]. Another patient on Clexane presented with ACG secondary to massive subchoroidal hemorrhage. Nevertheless, acute glaucoma precipitated by anticoagulation is a rare occurrence [25].

\section{Others}

Some miscellaneous causes of ACG will be covered in this section. Bilateral AACG was observed in a 29 year old lady who consumed ecstasy and marijuana. The mechanism proposed was that ecstasy increases the release of monoamine neurotransmitters such as serotonin, noradrenaline and dopamine, while inhibiting reuptake of serotonin. As a result, there is mydriasis which precipitates ACG [26]. Latanoprost, a medication used to treat glaucoma, has also been linked with two cases of AACG. Latanoprost is thought to induce ciliary muscle swelling which pushes the iris-diaphragm anteriorly and thus further predisposes the patient to AACG [27]. Methylsulfonylmethane, a dietary supplement, has also been linked to AACG. It possesses a sulfonyl moiety that gives it a similar structure to sulfa-based drugs, thereby triggering AACG in a similar manner, via choroidal and ciliary body effusion [28]. Gabapentin and pregabalin use in the year before diagnosis of AACG was also found to be statistically significant with a relative risk of 1.42 . Topiramate, which has been researched and found to induce AACG, increases GABAergic transmission. In this topiramate-induced AACG, idiosyncratic ciliochoroidal detachments and ciliary body edema caused anterior displacement of the lens-iris diaphragm [29].

There has been one report of cabergoline-induced bilateral AACG. Ultrasound biomicroscopy of the patient revealed choroidal thickening, effusion beneath the ciliary body and anterior rotation of the iris-ciliary body in each eye [30]. Daratumumab, an anti-CD38 monoclonal antibody used in multiple myeloma, was also found to induce bilateral AACG via a similar mechanism to Topiramate in a case report [31]. Administration of flucloxacillin and carbamazepine was also found to be the culprit in the case report of a 40 year old man presenting with bilateral AACG. Ultrasound biomicroscopy found uveal effusions, anterior rotation of the ciliary body and an anterior displacement of the iris-lens diaphragm, factors which all contribute to a narrower angle [32]. Botulinum toxin, a medication used widely in the cosmetic industry, can also be a precipitant. The proposed mechanism is its anticholinergic properties that produce a parasympathetic effect, inhibiting the pupillary sphincter and causing mydriasis. AACG was also observed in a patient treated with botulinum toxin for blepharospasm. The medication was injected periorbitally and thus it is presumed that the botulinum toxin diffused to the ciliary ganglion, inducing mydriasis [33].

A novel case of bilateral AACG was noted after tramadol was administered subcutaneously in a patient with bilateral open fractures of the femur [34]. Tramadol can cause miosis by stimulating the opioid receptors or cause mydriasis by stimulating the adrenergic receptors. However, it is more likely to cause 
mydriasis in a patient who is a poor metabolizer of tramadol, as there will be a delay in the conversion of the drug to its active metabolite. Apart from drugs, auto-immune conditions such as systemic lupus erythematosus (SLE) has also been associated with AACG. SLE can present with various manifestations of ocular disease, ranging from the more common keratoconjunctivitis sicca to the rarer optic neuritis. Choroidal disease is another rare complication of SLE. The associated choroidal effusion produces intraocular hypertension, thus potentially precipitating secondary ACG [35].

Head and orbital trauma, a very common presentation in the ED, was cited as the unusual cause of AACG in a 52-year-old lady who fell off a ladder [36]. This rise in intraocular pressure was potentially caused by traumatic hyphema that blocked the trabecular network and the canal of Schlemm, resulting in the buildup of aqueous humour. Mydriasis from the sympathetic stimulation as a result of the stress response to trauma could also have potentiated the AACG. One study also reported a unilateral AACG in a 65-year-old man undergoing a laminectomy that was suspected to have been caused by his prone position and the administration of mydriatic agents during the operation [37]. Lying prone in a dark room has previously been described as a provocative test for early detection of ACG in previous literature. This could possibly be due to the lens falling onto the iris in the prone position, increasing pupillary block and worsening the ACG [38]. Such mechanism is especially important to note in intubated patients who require prone oxygenation during this COVID-19 pandemic.

\section{Discussion}

Polypharmacy is a challenging healthcare issue globally. Geriatric patients in the United States for example, make up 13\% of the population but use $33 \%$ of all prescription drugs in the country. Polypharmacy is even more of an issue in nursing homes, where on average, residents are on 6 different drugs with $20 \%$ taking 10 or more drugs. No research has been done on the elderly in Singapore, but numbers are likely to be quite similar. With greater rates of polypharmacy, there is also a greater potential for unwanted adverse events. In Singapore, a study found that of all instances of polypharmacy in local nursing homes, the most prevalent medications were cardiovascular drugs (19.6\%), psychoactive drugs (13.9\%), gastrointestinal drugs (14.2\%), vitamins (15.9\%), antihistamines (4.7\%) and analgesia (6.4\%) [39]. Among all these drugs, there is a gigantic overlap with the culprit mentioned in the earlier sections that could potentially precipitate ACG. These include diuretics, SSRIs, H2 blockers and sedating antihistamines. With such a large proportion of the elderly on these types of drugs, we should be aware that polypharmacy may be a very possible risk factor for any cases of ACG encountered in the ED.

One study found that $18.6 \%$ of patients with primary ACG were found to have been prescribed drugs contraindicated for
PACG. $25 \%$ of patients with AACG had received contraindicated drugs for PACG. The most common drugs prescribed were benzodiazepine hypnotics which have anticholinergic properties, followed by adrenergic agonists used to treat overactive bladder. In the group who received drugs contraindicated for PACG, the median number of drug prescriptions was far higher than the group that did not receive the contraindicated drugs. The risks of prescribing contraindicated drugs such as anticholinergics should be made known amongst all healthcare professionals [40]. In addition, many drugs commonly administered in the ED have been reported to trigger AACG. A prospective study on common drugdrug interaction in an India ED found that the most common drugs prescribed were Ondansetron, Pantoprazole, Furosemide, Aspirin, Atorvastatin, Ceftriaxone and Heparin [41]. The drugs of note here are furosemide and heparin which were prescribed to 68 (43.58\%) and out of the 156 patients. As mentioned earlier, sulfa-based drugs such as furosemide can precipitate AACG via ciliary body edema and relaxation of lens zonules.

Another retrospective review of drug-drug interactions in an American ED also looked at the most common prescriptions over a 2 week period [42]. The most common prescriptions unsurprisingly included analgesics such as Ibuprofen (181 prescriptions), Oxycodone (92 prescriptions), Acetaminophen (71 prescriptions) and Aspirin (68 prescriptions). Additionally, Lorazepam (95 prescriptions), Corticosteroids (77 prescriptions), Diazepam (48 prescriptions), Metoprolol (36 prescriptions) and Albuterol (33 prescriptions) were also very common prescriptions in their ED. Atenolol, Cephalosporins and Ranitidine were tied for the 8th most common drug prescribed with 43 prescriptions each. Among all the drugs mentioned, a third can precipitate ACG, namely, Lorazepam, Diazepam, Ranitidine and Albuterol. Lorazepam and Diazepam are benzodiazepines with anticholinergic properties, Ranitidine is an antihistamine and Albuterol is an adrenergic. Several other ED drugs listed by the study that can precipitate ACG include Warfarin (14 prescriptions, Furosemide (12 prescriptions), Epinephrine (9 prescriptions), Diphenhydramine (7 prescriptions), Alprazolam (5 prescriptions), Gabapentin (5 prescriptions), Hydrochlorothiazide (4 prescriptions), Trimethoprim-sulfamethoxazole (3 prescriptions), Pseudoephedrine (2 prescriptions) and Hydroxyzine (2 prescriptions). Out of the 66 drugs prescribed over the 2 weeks, 16 drugs (24\%) can trigger ACG. There is clearly a large overlap among common drugs prescribed in the ED and drugs that have the potential to cause an angle closure attack.

\section{Conclusion}

Polypharmacy is a growing problem in healthcare due to the progressively aging population worldwide. Drugs that precipitate ACG are varied and most are commonly prescribed to these patients who are already on multiple medications. Coupled with the fact that 
many ED drugs have been shown to trigger ACG, this makes a case of drug-precipitated ACG in the ED very possible. Furthermore, if the patient is not in a state to give a proper history, AACG can easily be missed. Emergency physicians ought to have a heightened suspicion, especially when attending to patients with predisposing risk factors for ACG such as an elder, female patient with small stature.

\section{Declaration of Conflict of Interest}

The authors have no conflict of interest to disclose.

\section{References}

1. Prum BE Jr, Herndon LW Jr, Moroi SE, Mansberger SL, Stein JD, et al. (2016) Primary Angle Closure Preferred Practice Pattern $\left({ }^{\circledast}\right)$ Guidelines. Ophthalmology 123(1): P1-P40.

2. Flores-Sánchez BC, Tatham AJ (2019) Acute angle closure glaucoma. Br J Hosp Med (Lond) 80(12): C174-c179.

3. Bourne RR, Stevens GA, White RA, Smith JL, Flaxman SR, et al. (2013) Causes of vision loss worldwide, 1990-2010: a systematic analysis. Lancet Glob Health 1(6): e339-e349.

4. Lachkar Y, Bouassida W (2007) Drug-induced acute angle closure glaucoma. Curr Opin Ophthalmol 18(2): 129-133.

5. Weinreb RN, Aung T, Medeiros FA (2014) The pathophysiology and treatment of glaucoma: a review. Jama 311(18): 1901-1911.

6. Hilmer SN, Gnjidic D (2009) The effects of polypharmacy in older adults. Clin Pharmacol Ther 85(1): 86-88.

7. Yang MC, Lin KY (2019) Drug-induced Acute Angle-closure Glaucoma: A Review. J Curr Glaucoma Pract 13(3): 104-109.

8. Liew G, Mitchell P, Wang JJ, Wong TY (2006) Fundoscopy: to dilate or not to dilate? BMJ 332(7532): 3.

9. Belliveau AP, Somani AN, Dossani RH (2021) Pupillary Light Reflex. StatPearls. Treasure Island (FL).

10. Babineau MR, Sanchez LD (2008) Ophthalmologic procedures in the emergency department. Emerg Med Clin North Am 26(1): 17-34.

11. Nakakura S (2018) Icare $\left({ }^{\circledast}\right)$ rebound tonometers: review of their characteristics and ease of use. Clin Ophthalmol 12: 1245-1253.

12. Sacks D, Baxter B, Campbell BCV, Carpenter JS, Cognard C, et al. (2018) Multisociety Consensus Quality Improvement Revised Consensus Statement for Endovascular Therapy of Acute Ischemic Stroke. Int J Stroke 13(6): 612-632.

13. Schuster AK, Erb C, Hoffmann EM, Dietlein T, Pfeiffer N (2020) The Diagnosis and Treatment of Glaucoma. Dtsch Arztebl Int 117(13): 225234.

14. Sharts-Hopko NC, Glynn-Milley C (2009) Primary open-angle glaucoma. Am J Nurs 109(2): 40-47.

15. Amerasinghe N, Aung T (2008) Angle-closure: risk factors, diagnosis and treatment. Prog Brain Res 173: 31-45.

16. See JL, Aquino MC, Aduan J, Chew PT (2011) Management of angle closure glaucoma. Indian J Ophthalmol 59(1): S82-S87.

17. Tripathi RC, Tripathi BJ, Haggerty C (2003) Drug-Induced Glaucomas. Drug Safety 26(11): 749-767.

18. Geanon JD, Perkins TW (1995) Bilateral acute angle-closure glaucoma associated with drug sensitivity to hydrochlorothiazide. Arch Ophthalmol 113(10): 1231-1232.
19. Boundaoui ON, Woodruff TE (2016) Presumed Furosemide-associated Bilateral Angle-Closure Glaucoma. J Glaucoma 25(8): e748-e750.

20. Jain NS, Ruan CW, Dhanji SR, Symes RJ (2021) Psychotropic DrugInduced Glaucoma: A Practical Guide to Diagnosis and Management. CNS Drugs 35(3): 283-289.

21.Zormpas S, Matsou A, Antunes DM, Panos C (2021) Sumatriptan dose increase-induced acute angle closure glaucoma in chronic migraine sufferer. BMJ Case Rep 14(2): e235880.

22. Hsu C-R, Chen Y-H, Tai M-C, Lu D-W (2017) Sumatriptan-induced angleclosure glaucoma: A case report. Medicine 96(22): e6953.

23. Shen E, Farukhi S, Schmutz M, Mosaed S (2018) Acute Angle-closure Glaucoma Associated With Aripiprazole in the Setting of Plateau Iris Configuration. Journal of Glaucoma 27(2): e40-e43.

24. Schlote T, Freudenthaler N, Gelisken F (2005) Akutes Winkelblockglaukom nach massiver intraokularer Blutung bei exsudativer altersbedingter Makuladegeneration unter gerinnungshemmender Therapie. Der Ophthalmologe 102(11): 1090-1096.

25. Neudorfer M, Leibovitch I, Goldstein M, Loewenstein A (2002) Massive choroidal hemorrhage associated with low molecular weight heparin therapy. Blood Coagulation \& Fibrinolysis 13(3): 257-259.

26. Trittibach P, Frueh BE, Goldblum D (2005) Bilateral angle-closure glaucoma after combined consumption of "ecstasy" and marijuana. Am J Emerg Med 23(6): 813-814

27. Yalvac IS, Tamcelik N, Duman S (2003) Acute angle-closure glaucoma associated with latanoprost. Jpn J Ophthalmol 47(5): 530-531.

28. Hwang JC, Khine KT, Lee JC, Boyer DS, Francis BA (2015) Methylsulfonyl-methane (MSM)-induced acute angle closure. J Glaucoma 24(4): e28-e30.

29. Browne MJ, Zakrzewski H, Carleton B, Etminan M, Mikelberg FS (2019) Association of Gabapentin or Pregabalin Use and Incidence of Acute Angle-closure Glaucoma. J Glaucoma 28(9): 777-779.

30. Razmjoo H, Rezaei L, Dehghani A, Peyman A, Akhlaghi M (2011) Bilateral angle-closure glaucoma in a young female receiving cabergoline: a case report. Case Rep Ophthalmol 2(1): 30-33.

31. Edwards RG, Vanderhoof S, Palestine A, Seibold LK (2020) Bilateral Secondary Angle Closure During Daratumumab Infusion: A Case Report and Review of the Literature. J Glaucoma 29(8): e83-e86.

32. Chan KC, Sachdev N, Wells AP (2008) Bilateral acute angle closure secondary to uveal effusions associated with flucloxacillin and carbamazepine. Br J Ophthalmol 92(3): 428-430.

33. Ah-Kee EY, Egong E, Shafi A, Lim LT, Yim JL (2015) A review of druginduced acute angle closure glaucoma for non-ophthalmologists. Qatar Med J 2015(1): 6.

34. Mahmoud A, Abid F, Ksiaa I, Zina S, Messaoud R, et al. (2018) Bilateral acute angle-closure glaucoma following tramadol subcutaneous administration. BMC Ophthalmol 18(1): 50.

35. Štěpánková J, Kinštová L, Gažová I, Kodetová M, Cendelín J, et al. (2019) SIMULTANEOUS BILATERAL ACUTE ANGLE-CLOSURE GLAUCOMA IN MILLER FISHER SYNDROME. Cesk Slov Oftalmol 75(4): 210-218.

36. Tse DM, Titchener AG, Sarkies N, Robinson S (2009) Acute angle closure glaucoma following head and orbital trauma. Emerg Med J 26(12): 913.

37. Stewart RJ, Landy DC, Lee MJ (2016) Unilateral Acute Angle-Closure Glaucoma After Lumbar Spine Surgery: A Case Report and Systematic Review of the Literature. Spine (Phila Pa 1976) 41(5): E297-E299.

38. Hyams SW, Friedman Z, Neumann E (1968) Elevated intraocular pressure in the prone position. A new provocative test for angle-closure glaucoma. Am J Ophthalmol 66(4): 661-672. 
39. Mamun K, Lien CT, Goh-Tan CY, Ang WS (2004) Polypharmacy and inappropriate medication use in Singapore nursing homes. Ann Acad Med Singap 33(1): 49-52.

40. Tanaka F, Shibatani N, Fujita K, Ikesue H, Yoshimizu S, et al. (2011) Polypharmacy-associated potential contraindications of drug prescriptions in patients with primary angle closure disease in a realworld setting. Journal of Pharmaceutical Health Care and Sciences 7(1): 17.

ISSN: 2574-1241

DOI: 10.26717/BJSTR.2021.36.005878

Fatimah Lateef. Biomed J Sci \& Tech Res

(c) (P) This work is licensed under Creative BY Commons Attribution 4.0 Licens

Submission Link: https://biomedres.us/submit-manuscript.php
41. Barot PA, Malhotra SD, Rana DA, Patel VJ, Patel KP (2013) Drug utilization in emergency medicine department at a tertiary care teaching hospital: A prospective study. J Basic Clin Pharm 4(4): 78-81.

42. Goldstein J, Jaradeh I, Jhawar P, Stair T (2004) Drug-Drug Interactions: Frequency \& Type, Potential \& Actual, Triage \& Discharge. The Internet Journal of Emergency and Intensive Care Medicine 8(2).

$\begin{array}{ll}\text { BIOMEDICAL } & \text { Assets of Publishing with us } \\ \text { RESEARCHES } & \text { - Global archiving of articles } \\ \text { - Immediate, unrestricted online access }\end{array}$

